\title{
THE
}

2017

\section{DNA-Carbon Nanotube Complexation Affinity and Photoluminescence Modulation Are Independent}

\author{
Prakrit V. Jena \\ Mohammad M. Safaee \\ University of Rhode Island \\ Daniel A. Heller \\ Daniel E. Roxbury \\ University of Rhode Island, roxbury@uri.edu \\ Follow this and additional works at: https://digitalcommons.uri.edu/che_facpubs \\ Part of the Chemical Engineering Commons
}

The University of Rhode Island Faculty have made this article openly available.

Please let us know how Open Access to this research benefits you.

This is a pre-publication author manuscript of the final, published article.

Terms of Use

This article is made available under the terms and conditions applicable towards Open Access Policy Articles, as set forth in our Terms of Use.

\section{Citation/Publisher Attribution}

Prakrit, P. V., Safaee, M. M., Heller, D. A., \& Roxbury, D. (2017). DNA-Carbon Nanotube Complexation Affinity and Photoluminescence Modulation Are Independent. ACS Appl. Mater. Interfaces, 9(25), 21397-21405. doi: 10.1021/acsami.7b05678

Available at: http://dx.doi.org/10.1021/acsami.7b05678 


\title{
DNA-Carbon Nanotube Complexation Affinity and Photoluminescence
}

\section{Modulation Are Independent}

Prakrit V. Jena ${ }^{1}$, Mohammad M. Safaee ${ }^{2}$, Daniel A. Heller ${ }^{1,3}$, Daniel Roxbury ${ }^{2 *}$

${ }^{1}$ Memorial Sloan Kettering Cancer Center, New York, NY 10065

${ }^{2}$ Department of Chemical Engineering, University of Rhode Island, Kingston, RI 02881

${ }^{3}$ Weill-Cornell Medical College, New York, NY 10065

KEYWORDS. nanobiotechnology, near-infrared fluorescence, optical nanosensors, supramolecular absorption

\begin{abstract}
Short single-stranded DNA (ssDNA) has emerged as the natural polymer of choice for noncovalently functionalizing photoluminescent single-walled carbon nanotubes. In addition, specific empirically identified DNA sequences can be used to separate single species (chiralities) of nanotubes with exceptionally high purity. Currently, only limited general principles exist for designing DNA-nanotube hybrids amenable to separation processes, due in part to an incomplete understanding of the fundamental interactions between a DNA sequence and a specific nanotube structure, while even less is known in the design of nanotube-based sensors with determined optical properties. We therefore developed a combined experimental and analysis platform, based on time-resolved near-infrared fluorescence spectroscopy, to extract the complete set of photoluminescence parameters that characterize DNA-nanotube hybrids. Here, we systematically investigated the affinity of the $\mathrm{d}(\mathrm{GT})_{\mathrm{n}}$ oligonucleotide family for structurally-defined carbon nanotubes by measuring photoluminescence response of the nanotube upon oligonucleotide
\end{abstract}


displacement. We found, surprisingly, that the rate of displacement of oligonucleotides is independent of the coverage on the nanotube, as inferred through intrinsic optical properties of the hybrid. The kinetics of intensity modulation are essentially single exponentials, and the time constants, which quantify the stability of DNA binding, span an order of magnitude.

Surprisingly, these time constants do not depend on the intrinsic optical parameters within the hybrids, suggesting that DNA-nanotube stability is not due to increased nanotube surface coverage by DNA. Further, a principal component analysis of the excitation and emission shifts, along with intensity enhancement at equilibrium accurately identified the $(8,6)$ nanotube as the partner chirality to (GT)6 ssDNA. Combined, the chirality-resolved equilibrium and kinetics data can guide the development of DNA-nanotube pairs with tunable stability and optical modulation. Additionally, this high-throughput optical platform could function as a primary screen for mapping the DNA-chirality recognition phase space.

\section{Introduction}

The bandgap near-infrared (NIR) photoluminescence from semiconducting single-walled carbon nanotubes (SWCNT) is photostable, tunable, and sensitive to the local environment. ${ }^{1}$ Over the last 15 years, significant progress has been made in applying carbon nanotube photoluminescence towards a range of in vitro and in vivo biomedical applications. ${ }^{2}$ Efforts to synthesize nanotubes of a specific structure (chirality), ${ }^{3}$ along with methods to separate specific nanotube chiralities have achieved notable successes. ${ }^{4}$

For both biological applications and separation procedures, short single-stranded DNA plays an important role. Pristine single-walled carbon nanotubes require non-covalent functionalization to 
enable aqueous suspension while still retaining their intrinsic NIR optical properties. ${ }^{1}$ Additionally, separation or sensing applications that require a specific biomolecular interaction on the nanotube surface depend on the functionalizing polymer to impart a recognition ability. ${ }^{1}$ Short single-stranded DNA was initially identified as a polymer that could efficiently solubilize single-walled carbon nanotubes via non-covalent interactions forming DNA-nanotube hybrids. ${ }^{5}$ Subsequently, reports have shown that DNA facilitates separation of nanotube chiralities by imparting distinct hydrophobicities and/or charge densities on nanotubes by the specific interactions between certain sequences of DNA and specific $(\mathrm{n}, \mathrm{m})$ nanotube species. ${ }^{6-7}$ Additionally, this DNA sequence-nanotube chirality matching, hypothesized to be the result of extended hydrogen bonding networks amongst adsorbed nucleobases, ${ }^{6}$ inhibits oxygen induced quenching of the nanotube photoluminescence. ${ }^{8}$ Similarly, screening DNA-chirality pairs for analyte specificity ${ }^{9}$ led to the development of optical biosensors for a range of small molecule analytes. ${ }^{10}$ The ability to integrate molecular recognition into DNA-nanotube hybrids has been achieved via base-pair hybridization, ${ }^{11}$ antibody-coupling, ${ }^{12}$ and oligonucleotide aptamers. ${ }^{13}$

Several techniques have been employed to probe the fundamental interactions between DNA and the carbon nanotube surface. Absorption spectroscopy assays ${ }^{14-16}$ can be used to extract thermodynamic parameters, but are limited in their ability to fully resolve multiple chiralities. Modalities such as atomic force microscopy ${ }^{17}$ and thermogravimetric analysis are quantitative, ${ }^{18}$ but also cannot resolve nanotube chiralities. Techniques, including single molecule force spectroscopy to directly measure the force required to separate ssDNA from a nanotube ${ }^{19}$ and nanoparticle fluorescence spectroscopy to observe DNA-nanotube interactions in a microfluidic cell, ${ }^{20}$ continue to further out understanding. Molecular dynamics simulations can be used to visualize the DNA structure on a nanotube with unprecedented resolution, ${ }^{21}$ but simulations are 
costly in time and limited to simple model systems. ${ }^{22}$ In summary, the techniques currently available are either unable to resolve a sufficient number of individual chiralities or cannot be scaled to represent ensemble averaged data. Therefore, our understanding of the DNA-nanotube interactions at their interface and the resulting optical signal transduction remains incomplete.

The optical transition energies of carbon nanotubes are modulated by the local environment, ${ }^{23}$ with the local dielectric constant ${ }^{24}$ influencing both quantum yields and solvatochromic shifts. ${ }^{25}$ For DNA-carbon nanotube hybrids, the microenvironment of the nanotube is a complex equilibrium structure that depends on DNA sequence, ${ }^{26}$ water density,${ }^{27}$ and nanotube chirality. ${ }^{22}$ A recent technique to study the stability of DNA-nanotubes by competing ssDNA with a surfactant ${ }^{20}$ highlighted the potential of time-resolved spectroscopy to directly measure both optical transition energies and the kinetics of DNA desorption. While these assays were limited to physically-separated single nanotube chiralities, optical advancements such as real time hyperspectroscopy ${ }^{28}$ allow for spectral separation of individual chiralities in an unsorted sample. By directly measuring equilibrium optical transitions and transient intensity modulations, we could address unanswered questions in the literature, such as: Is DNA stability a function of DNA sequence length, or nanotube chirality, or both? Does the degree of nanotube surface coverage by DNA determine the stability of the DNA-nanotube hybrid, and how does this answer depend on the nanotube chirality? Can optical signatures help screen and identify target chiralities for separation by DNA sequences?

Here, we examined DNA-nanotubes for their binding affinities and optical modulations, using a combination of photoluminescence excitation/emission (PLE) plots and time-resolved nearinfrared photoluminescence spectroscopy. We found that stability of DNA on a nanotube could not be predicted from equilibrium photoluminescence modulations, but required a kinetics 
measurement via a surfactant induced DNA displacement. The kinetics of DNA displacement were single exponential, with time constants that spanned an order of magnitude. Interestingly, these time constants were essentially independent of nanotube surface coverage, as inferred by relative excitation/emission energies, suggesting that the strength of DNA binding is the primary determinant of stability of the DNA-nanotube hybrid. We propose that our high throughput spectroscopy and analysis method can screen and identify DNA-chirality pairs which optimize photoluminescence response and stability for both sensing and separation applications.

\section{Results and Discussion}
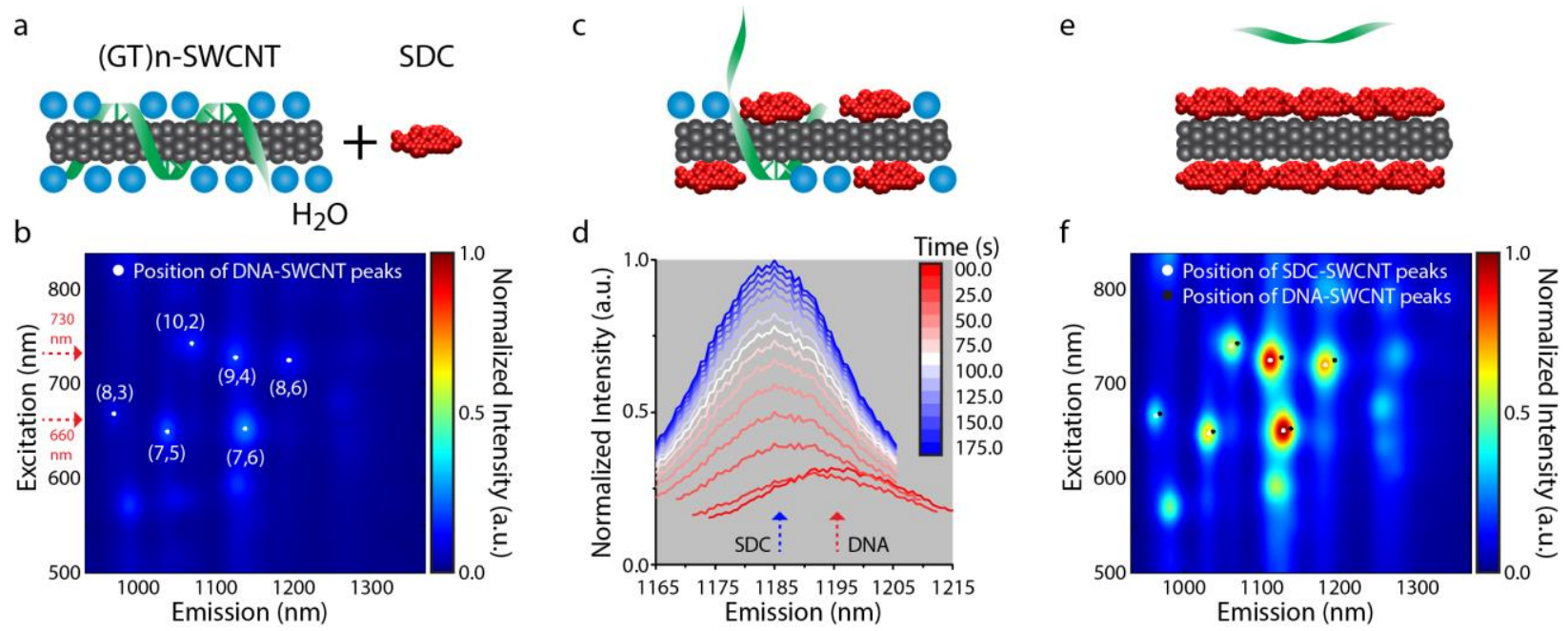

Figure 1. Schematic of the surfactant-induced DNA displacement mechanism and corresponding optical output. (a) Equilibrium conformation of DNA on a nanotube, and the (b) corresponding optical parameters. (c) Addition of SDC changes the emission spectra (d) in time. (e) The equilibrium SDC-SWCNT and the (f) corresponding optical parameters.

To systematically investigate DNA-nanotube interactions as a function of nanotube chirality and DNA-sequence length, we prepared $\operatorname{ss}(\mathrm{GT})_{\mathrm{n}}$-nanotube hybrids where $\mathrm{n}=3,6,9,12,15$, or 30 repeats. As apparent from the high peak-to-valley ratios and narrow bands in the absorption spectra (Fig. S1), regardless of DNA sequence length, the entire mixture of HiPco nanotubes were well dispersed. For the equilibrium conformation of $\mathrm{ss}(\mathrm{GT})_{\mathrm{n}}$-nanotubes (Fig. 1a), we 
obtained the intensity, excitation, and emission peaks for the $(8,3),(7,5),(10,2),(9,4),(7,6)$ and $(8,6)$ chiralities, from the initial photoluminescence excitation/emission (PLE) plots (Fig. 1b).
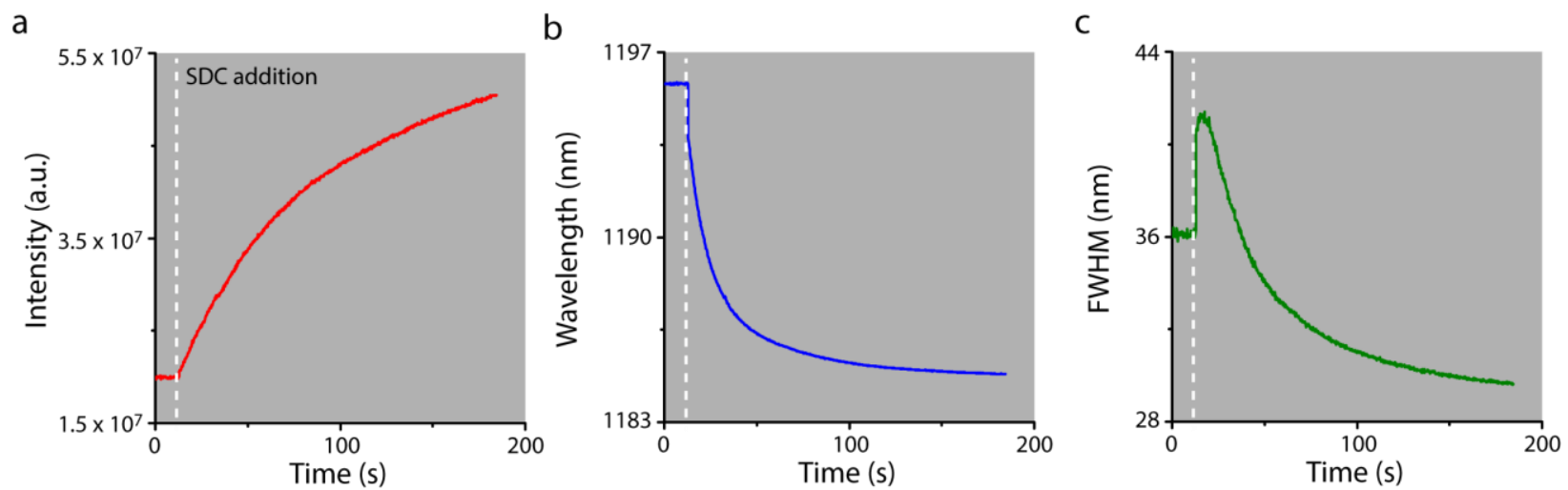

Figure 2. Modulation of emission parameters as a function of time. (a) Intensity, (b) center wavelength and (c) full-width at half-maximum of the $(\mathrm{GT})_{30}-(8,6)$ hybrid following SDC addition.

We then added excess sodium deoxycholate (SDC), known to displace the DNA from the nanotube surface. ${ }^{29}$ On adding SDC (0.1\% final concentration), we observed the dynamic process of surfactant binding to the exposed nanotube surface (Fig. 1c) and the resultant modulation in the nanotube emission spectra (Fig. 1d) upon excitation at either $660 \mathrm{~nm}$ or 730 nm (red arrows in Fig. 1a). At equilibrium, the DNA initially adsorbed on each nanotube chirality is completely displaced by SDC (Fig. 1e), demonstrated by the convergence of all optical transition wavelengths (Fig. S2) obtained from the final PLE plots (Fig. 1f). When compared with the excitation and emission peaks from SDC-nanotubes (i.e. SWCNT dispersed directly in SDC), we find that the DNA-nanotube spectra on SDC addition are as blue-shifted or more blue-shifted than the corresponding SDC-nanotube spectra. This discrepancy is likely due to the differential ability of SDC and DNA to singly exfoliate the nanotube samples. ${ }^{30}$ The resulting data of photoluminescence modulation of DNA-to-SDC replacement on the nanotube surface are presented in Table S1. 
The evolving emission spectra (Fig. 1d) is an ensemble average of nanotubes in solution, with each nanotube containing sections with either DNA or SDC coverage. When fit with a Lorentzian profile, ${ }^{31}$ we extracted peak intensity (Fig. 2a), center wavelength (Fig. 2b) and fullwidth-at-half-maximum (FWHM, Fig. 2c) as a function of time, for each of the 36 sequencechirality pairs (Figs. S3 and S4). Interestingly, certain DNA-chirality pairs (e.g. (GT)6-(10,2)) exhibit a large initial blue-shift followed by slow red-shift to an equilibrium value. We treated each emission spectrum (Fig. S5a) as a linear combination of the DNA-only and SDC-only spectrum $^{29}$ (Fig. S5b), and fit all transient spectra (Fig. S5c) as the sum of these two components (Fig. S5d). The anti-correlated contributions of the DNA and SDC components, plotted as a function of time (Fig. S5e), suggest a concomitant decrease in DNA coverage and increase in SDC coverage. However, as the blue-shifted SDC-peak is significantly ( $\sim$-fold) brighter than the DNA peak, the apparent wavelength shift is faster than the intensity increase (Fig. S5f). We observed this phenomenon in the experimental data for every sequence-chirality pair (Figs S3 and S4). For the rest of our analysis, we used the peak intensity at the SDC-emission wavelength as a metric for the degree of DNA displacement due to replacement by SDC. 

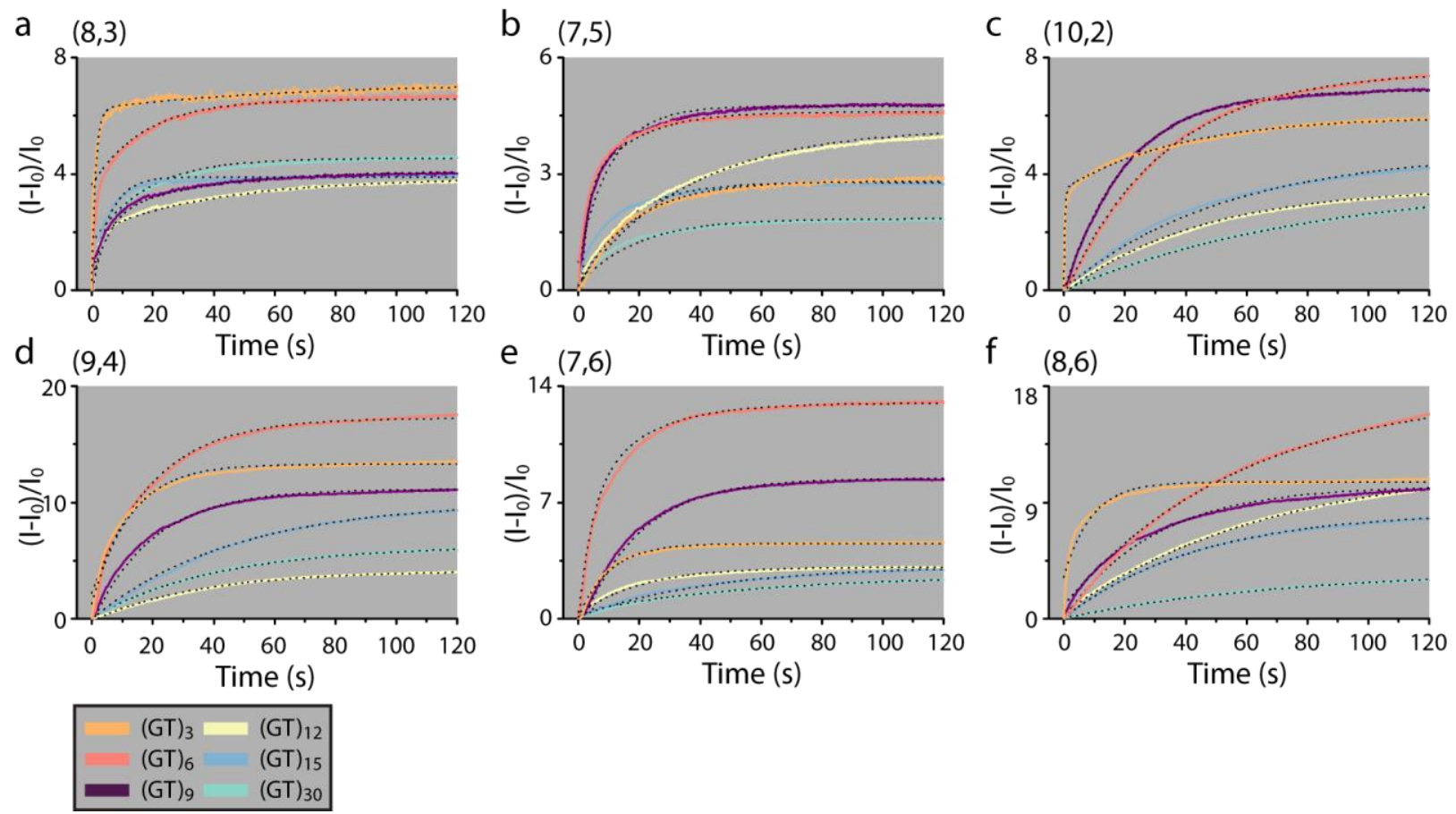

Figure 3. Fractional intensity increase (of the SDC peak) as a function of time for all (GT)n sequences for the (a) $(8,3)$, (b) $(7,5)$, (c) $(10,2)$, (d) $(9,4),(e)(7,6)$ and the (f) $(8,6)$ chirality. Dotted lines indicate the exponential fits for each sample.

As a relative measure of the DNA-nanotube hybrid stability, we plotted the emission intensity of the SDC-peak wavelength as a function of time, for each DNA sequence-nanotube chirality pair. The majority (30/36) time traces of the fractional increase in intensity (Fig. 3) were fit with a single exponential association (Table $\mathrm{S} 2, \mathrm{r}^{2}>0.95$ for all time traces). However, traces for $(8,3)$ and for the shorter sequences (6/36 in total) were significantly better fit as a sum of two exponentials, suggesting a more complicated two-stage mechanism for these DNA-chirality pairs (Table S3). Dashed lines in Fig. 3 represent the best fits (single or double exponential) for each sequence-chirality pair. Physically, the processes occurring on the nanotube surface include (1) binding of SDC to the exposed nanotube surface, (2) rearrangement and displacement of DNA by SDC, and (3) SDC reorganization and micelle formation over the nanotube surface. We propose that the separate rates of the above processes might be too fast to experimentally observe 
(and at SDC concentrations above $0.1 \%$, even the single exponential behavior was too fast to fit within the experimental time resolution of $0.5 \mathrm{~s}$ ). Future studies, including titrating the SDC concentration to develop a Langmuir adsorption model, or performing the kinetics measurements at lower temperatures or lower SDC concentrations, could provide further insight into the mechanisms being investigated.

For comparing across samples, we used the time constants from single exponential fits as an experimentally derived metric for DNA-nanotube hybridization affinity. The combined kinetics and PLE data provide ten descriptors of each sequence-chirality pair that can be used to assess stability and dynamic range in optical responses, respectively, in DNA-nanotube hybrids.

We asked if photoluminescence parameters and their modulations could predict sequencechirality recognition pairs and potentially enable optical screening to identify target chiralities for specific DNA sequences. We thus assessed excitation shifts, emission shifts, and intensity enhancements for all DNA-chirality pairs and compared visually using heat maps (Fig. 4a-c). Certain trends were visible in the data, such as the large excitation shift and intensity enhancement for $(8,6)$, and the maximum emission shift for $(9,4)$. We systematically looked for correlations between the five unique photophysical parameters, both within individual chiralities and across the entire nanotube population. As all sequence-chirality pairs converged to the same SDC-coated nanotube sample (Fig. S2), the initial excitation and emission peaks shifted to the same value. To our surprise, intensity enhancement was not correlated to either excitation or emission shift for any chirality, and only the $(8,6)$ showed a statistically significant dependence between the excitation and emission energy shifts (Fig. S6). However, excitation energy shifts and emission energy shifts were weakly correlated with intensity enhancement at the population 
level ( 0.369 and 0.395 Spearman correlation respectively, $\mathrm{p}<0.05$ for both), suggesting a potential connection between the DNA sequence family and the nanotube chirality.
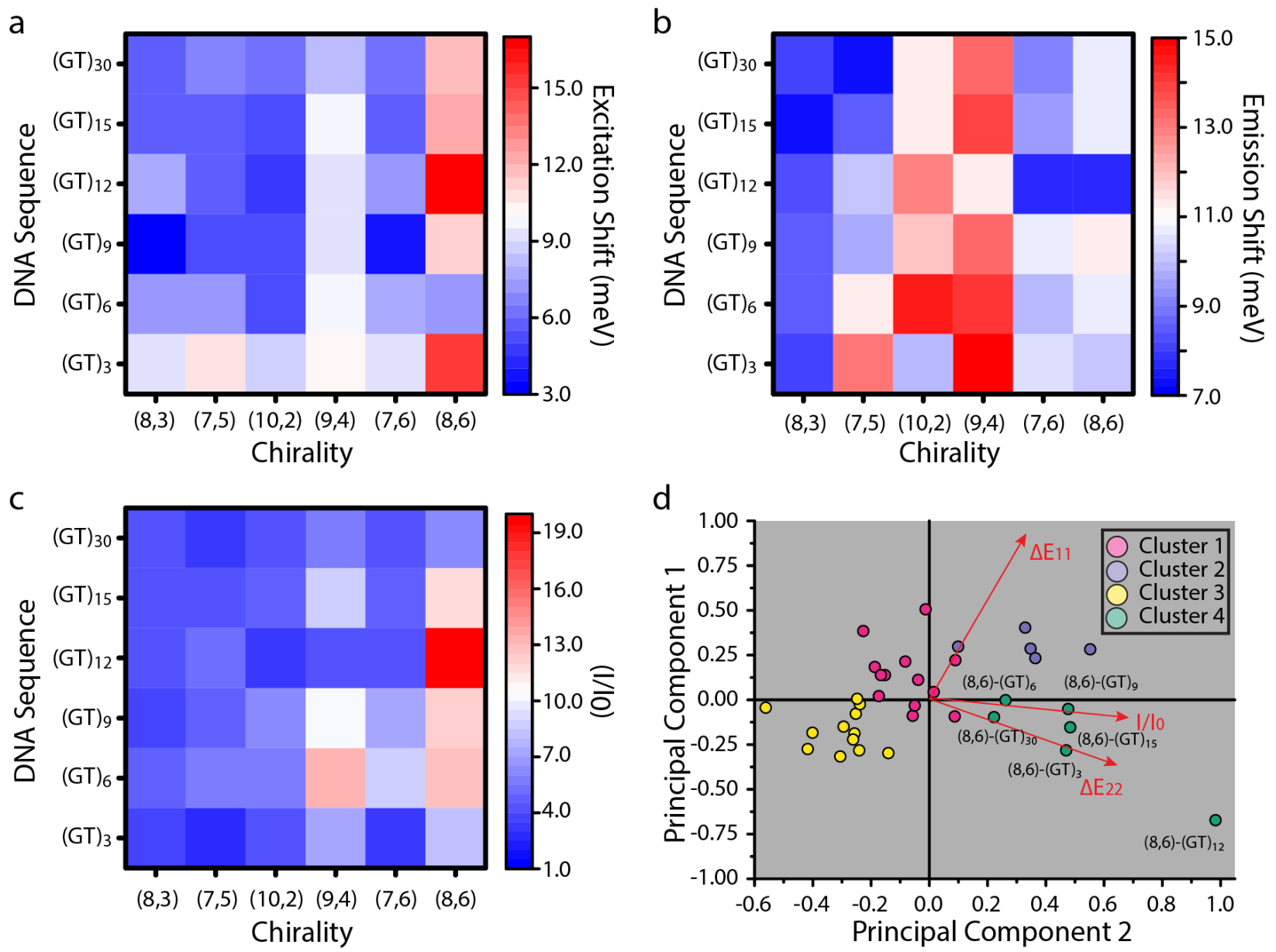

Figure 4. Heat map, as a function of nanotube chirality and DNA sequence, of the (a) excitation energy shifts, (b) emission energy shifts and the (c) fractional intensity increases. The nanotube chiralities are arranged in order of decreasing bandgap energy. (d) Principal component analysis of the excitation shift, emission shift, and intensity enhancement, for the first two principal components.

To investigate the sequence-chirality connection to these optical parameters, we used principal component analysis on the excitation shift, emission shift, and intensity enhancement data for the DNA sequence-nanotube chirality pairs. From the three principal components, over $87 \%$ of the variance in the data could be explained by the first two. A scatter plot of the first two principal 
components maximizes the variance in the data while reducing the dimensionality from three to two. We asked whether the DNA sequence-nanotube chirality pairs in the principal component phase space clustered in a meaningful way. A two-dimensional k-means algorithm (without any initial guesses) identified four distinct clusters (Fig. 4d). ${ }^{32}$ Interestingly, one cluster (labeled 4 in Fig. 4d) was composed entirely of all six ss(GT) $)_{n}-(8,6)$ pairs (Table S4). Though no optical parameter exclusively selects the $(8,6)$ chirality, k-means analysis of the principal components differentiates the optical response of all $(8,6)$ chirality nanotubes from the other 30 DNA sequence-nanotube chirality pairs. As ss $(\mathrm{GT})_{6}$ was initially identified as the recognition sequence for the $(8,6)$ chirality using ion-exchange chromatography, ${ }^{6}$ the identification of $(\mathrm{GT})_{\mathrm{n}}-(8,6)$ nanotubes as a distinct cluster in principal component analysis is a promising result. Though photoluminescence measurements of the addition of small molecules to polymer-nanotube hybrids have highlighted the complexity of the interactions, ${ }^{33-34}$ the data suggests that our method, validated for one specific DNA-sequence family and chirality, could potentially provide a scalable and predictive approach to determining candidate DNA recognition sequences for specific nanotube chiralities.

To characterize DNA-nanotube hybridization affinities, we generated a heat map of the time constants as a function of nanotube chirality and DNA sequence length (Fig. 5a). Visually apparent, the heat map indicated a general trend, with longer time constants more prevalent amongst DNA-nanotube hybrids with longer DNA and larger diameter nanotubes. When mapped in ascending order, the time constants (larger time constant indicating a stronger binding affinity of DNA to the nanotube) increased from 5 to 80 seconds (Fig. 5b). A one-dimensional k-means analysis identified three classes of samples - least stable (time constants $<25 \mathrm{~s}$ ), stable $(25 \mathrm{~s}<$ time constants $<50 \mathrm{~s}$ ) and a small subset that are exceptionally stable (time constant $>50 \mathrm{~s}$ ). 
Interestingly, ss(GT) ${ }_{12}-(8,6)$ and ss $(\mathrm{GT})_{6}-(8,6)$ stand out as outliers, with high DNA-nanotube affinity despite the comparatively short DNA sequence length.

a
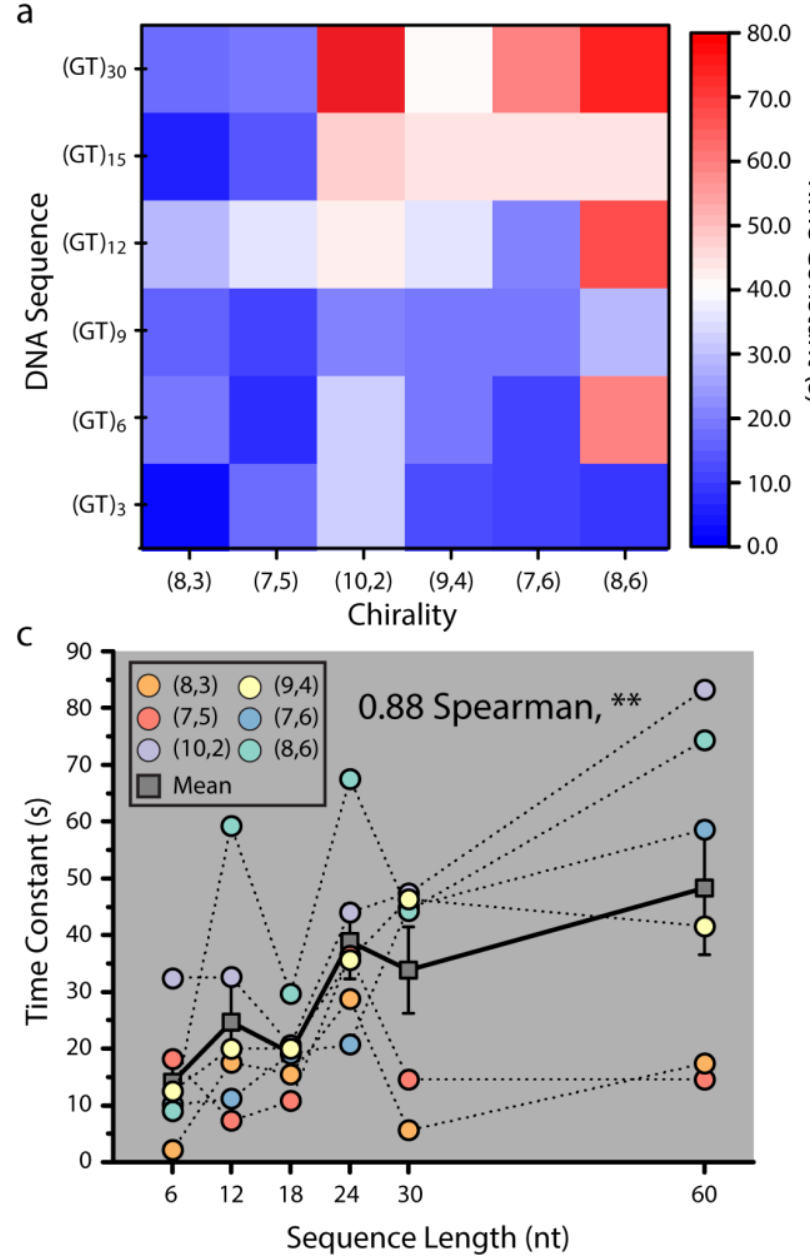

b

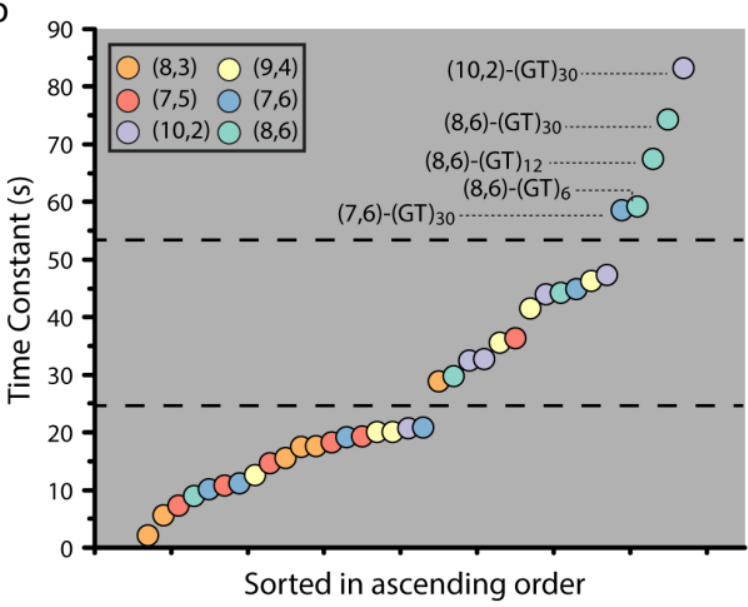

d

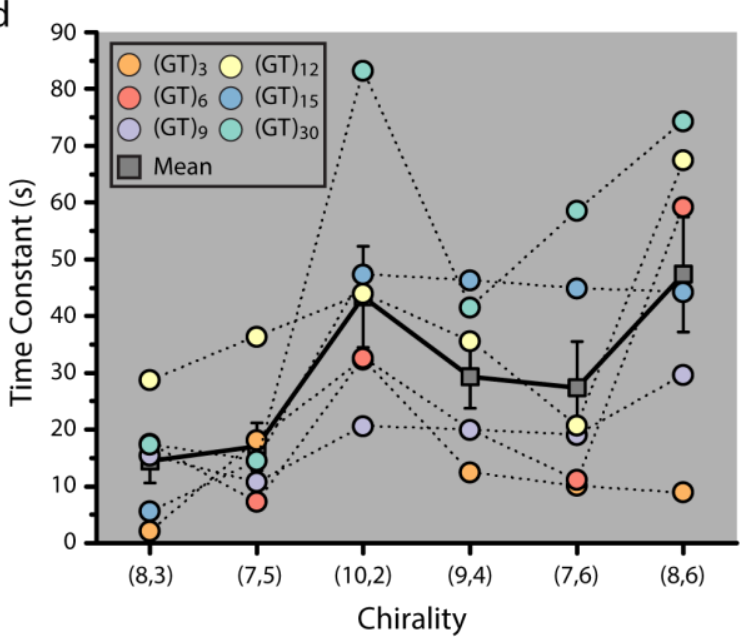

Figure 5. DNA stability as a function of DNA-SWCNT parameters (a) Heat map of single exponential time constant of fractional intensity increase as a function of DNA sequence length and chirality. (b) Time constants plotted in ascending order and color-coded by chirality. (c) Mean time constant (gray squares) as a function of DNA sequence length, with data points for each chirality represented as color-coded circles. (d) Mean time constant (gray squares) as a function of chirality, with data points for each DNA sequence represented as color-coded circles. Error bars are the s.e.m. across the contributing data points.

Next, we analyzed the time constants as a function of either sequence length or chirality. For the combined nanotube population, the stability of the $\mathrm{ss}(\mathrm{GT})_{\mathrm{n}}$ DNA correlated strongly with sequence length (Fig. 5c). However, the chirality-resolved data indicated this relationship to only 
be true for three out of six chiralities (Fig. S7). The time constants for each DNA sequence length did not correlate with nanotube chirality either (Fig. 5d), and the range of time constants across the six chiralities was large. Based on these results, we propose that DNA-nanotube binding affinity is strongly dependent on the specific interaction between a nanotube chirality and the DNA sequence, with no obvious systematic dependence on either. In the most striking example, $\operatorname{ss}(\mathrm{GT})_{3}-(9,4)$ and ss $(\mathrm{GT})_{30}-(7,5)$ had essentially identical time constants $(12.48 \mathrm{~s}$ vs $14.63 \mathrm{~s}$, respectively), despite a 54-base sequence length advantage for ss(GT) $)_{30}-(7,5)$. Even within a single chirality, such as the $(8,3), \operatorname{ss}(\mathrm{GT})_{3}-(8,3)$ and $\mathrm{ss}(\mathrm{GT})_{30}-(8,3)$ had statistically identical time constants $(\sim 17.5 \mathrm{~s})$. This analysis suggests that increased hybrid stability is not solely determined by the length of DNA sequence.

As the displacement of DNA by SDC on the nanotube surface induces a change in the NIR photoluminescence, we asked whether photoluminescence parameters correlated with the stability directly measured via DNA displacement kinetics. We hypothesized that the stability for a specific sequence-chirality pair could result from the extent of nanotube surface coverage by DNA. A nanotube with low DNA coverage would provide a larger and more accessible surface for oxygen and water molecules, when compared to a nanotube with high DNA coverage (Fig. 6a). Because water increases the local dielectric constant around the nanotube, ${ }^{23-25}$ a more stable DNA sequence-nanotube chirality pair with low surface exposure to water (high DNA coverage) should have excitation and emission peaks that correspond to higher energy optical transitions. ${ }^{26-}$ ${ }^{27}$ Instead, scatter plots of the excitation and emission energy peaks showed no correlation with the time constants, for any of the six chiralities (Fig. 6b-c). The intensity enhancement, often used as a proxy for nanotube surface coverage, ${ }^{10,20}$ also did not correlate with the time constants (Fig. 6d). No correlation was observed between photoluminescence parameters and DNA 
sequence length either (Fig. S8). As DNA stability on the nanotube does not appear to correspond to the relative excitation and emission peak positions, we conclude that DNA surface coverage is not the determining factor for DNA-nanotube hybridization stability. We propose that the strong binding observed between certain DNA sequences and nanotube chiralities is likely due to specific DNA conformations (Table 1), which has been suggested to enhance hydrogen bonding between DNA bases. ${ }^{6,22}$

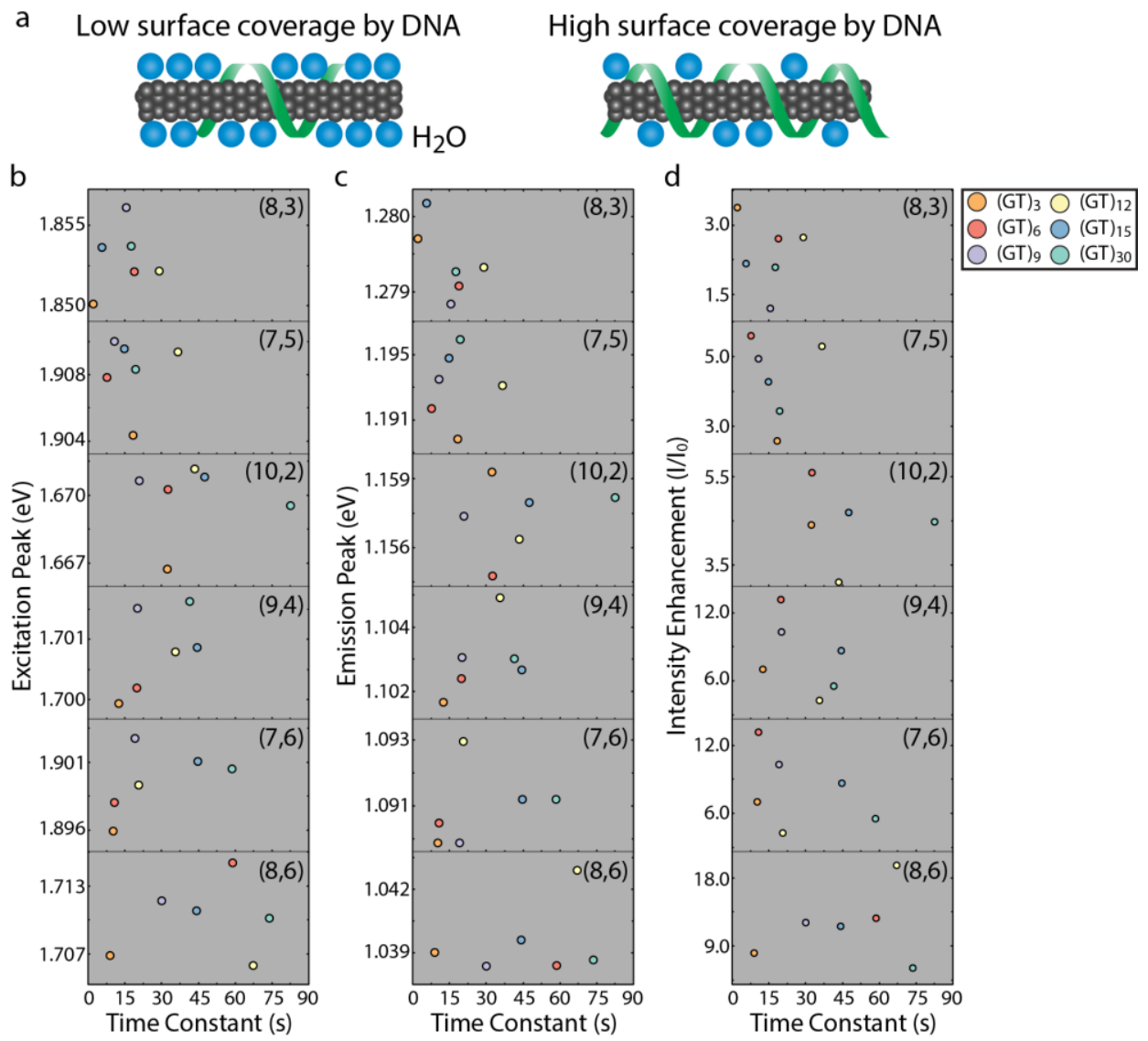

Figure 6. (a) Schematic for a DNA-nanotube with low DNA coverage and high DNA coverage. (b) Initial excitation peak, (c) initial emission peak and (d) intensity enhancement as a function of time constant for all DNA-nanotube samples, segmented by chirality. 
Table 1. Correlations between photoluminescence parameters and sequence length. Correlations presented as (Spearman Correlation, significance)

\begin{tabular}{|c|c|c|c|c|c|c|c|}
\hline \multirow[t]{2}{*}{ Parameters Compared } & \multicolumn{7}{|c|}{ Nanotube Chirality } \\
\hline & $(8,3)$ & $(7,5)$ & $(\mathbf{1 0 , 2 )}$ & $(9,4)$ & $(7,6)$ & $(8,6)$ & Combined \\
\hline $\begin{array}{l}\text { Sequence Length vs } \\
\qquad / / I_{0}\end{array}$ & - & - & - & - & - & - & - \\
\hline $\begin{array}{l}\text { Sequence Length vs } \\
\text { Initial Excitation }\end{array}$ & - & - & - & $\begin{array}{l}0.83 \\
0.040\end{array}$ & - & - & - \\
\hline $\begin{array}{l}\text { Sequence Length vs } \\
\text { Initial Emission }\end{array}$ & - & $\begin{array}{c}0.94 \\
0.0048\end{array}$ & - & - & - & - & - \\
\hline $\begin{array}{l}\text { Time Constant vs } \\
\text { Sequence Length }\end{array}$ & - & - & $\begin{array}{l}0.83 \\
0.042\end{array}$ & $\begin{array}{l}0.89 \\
0.019\end{array}$ & $\begin{array}{l}1, \\
0\end{array}$ & - & $\begin{array}{c}0.53 \\
0\end{array}$ \\
\hline $\begin{array}{c}\text { Time Constant vs } \\
\text { Intensity Enhancement }\end{array}$ & - & - & - & - & - & - & - \\
\hline $\begin{array}{l}\text { Time Constant vs } \\
\text { Initial Excitation }\end{array}$ & - & - & - & - & - & - & $\begin{array}{c}-0.41 \\
0.02 \\
\end{array}$ \\
\hline $\begin{array}{l}\text { Time Constant vs } \\
\text { Initial Emission }\end{array}$ & - & - & - & - & - & - & $\begin{array}{l}-0.51 \\
0.013\end{array}$ \\
\hline $\begin{array}{c}\text { Intensity Enhancement vs } \\
\text { Excitation Shift }\end{array}$ & - & - & - & - & - & - & $\begin{array}{l}0.37 \\
0.027\end{array}$ \\
\hline $\begin{array}{c}\text { Intensity Enhancement vs } \\
\text { Emission Shift }\end{array}$ & - & - & - & - & - & - & $\begin{array}{l}0.39 \\
0.017\end{array}$ \\
\hline $\begin{array}{l}\text { Excitation Shift vs } \\
\text { Emission Shift }\end{array}$ & - & - & - & - & - & $\begin{array}{l}-0.94 \\
0.048 \\
\end{array}$ & - \\
\hline $\begin{array}{l}\text { Initial Excitation vs } \\
\text { Initial Emission }\end{array}$ & - & - & - & - & - & - & - \\
\hline
\end{tabular}

Values presented are Spearman correlation coefficient and significance

\section{Conclusions}

We have developed a framework for experimentally determining both the stability and optical modulation of DNA-nanotube hybrids. By all indications, DNA-nanotube stabilities and photoluminescence modulations are essentially independent (Fig. 7). In the assay presented, equilibrium and dynamic measurements of DNA displacement from the nanotube surface by a surfactant allow both properties to be observed simultaneously. The DNA displacement follows single exponential kinetics, with time constants that range over an order of magnitude. Surprisingly, the stability appears to depend on the molecular interactions between a DNA sequence and a specific nanotube chirality, with no systematic dependence on DNA sequence length or chirality. Optical parameters that reflect accessibility of the solution to the nanotube 
surface do not correlate with the stability of the DNA-nanotube hybrid, suggesting that stability does not result from an increase in DNA coverage of the nanotube surface. However, principal components analysis and k-means clustering of optical parameters can identify the nanotube chirality with an enhanced affinity for a DNA family. As a result, predictions that are tailored for specific biological applications can be made. For instance, a low-stability but high-dynamic response DNA-nanotube pair like the $\operatorname{ss}(\mathrm{GT})_{3}-(9,4)$ hybrid can be selected for detecting amphiphilic proteins that displace DNA. Alternatively, a high-stability, high-dynamic response like the $\mathrm{ss}(\mathrm{GT})_{6}-(8,6)$ pair may be suitable for long-term monitoring of analytes in biological samples. We envision our optical screening approach enhancing the study of fundamental DNAnanotube interactions, and in the rational design of DNA-nanotube sensors for a variety of biological applications. The ability to optically identify recognition sequences for specific nanotube chiralities is another potentially valuable application.

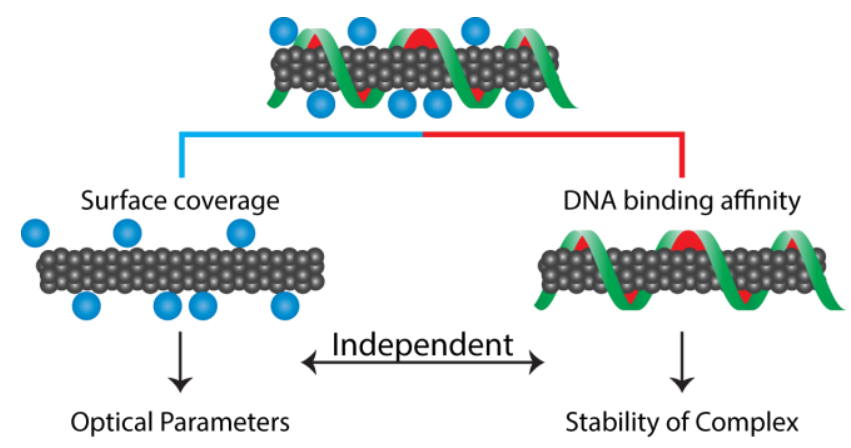

Figure 7. Model for photoluminescence parameters and hybridization affinities in DNAnanotube hybrids.

\section{Methods}

Nanotube Sample Preparation. HiPco single walled carbon nanotubes (Unidym, HiPco Raw) were suspended with DNA in $1 \mathrm{~mL}$ of deionized water with $100 \mathrm{mM} \mathrm{NaCl}$ (Sigma- 
Aldrich) by adding $1 \mathrm{mg}$ raw nanotubes to $2 \mathrm{mg}$ of desalted $\mathrm{ss}(\mathrm{GT}) \mathrm{n}(\mathrm{n}=3,6,9,12,15$, or 30$)$ oligonucleotide (Integrated DNA Technologies) in a microcentrifuge tube. The mixtures were ultrasonicated using a 1/8" tapered microtip (Sonics \& Materials, Sonics Vibracell) for $30 \mathrm{~min}$ at $40 \%$ amplitude, with an average power output of 8 Watts, in a $0{ }^{\circ} \mathrm{C}$ temperature-controlled microcentrifuge tube holder. After sonication, the dispersion was ultracentrifuged (Sorvall

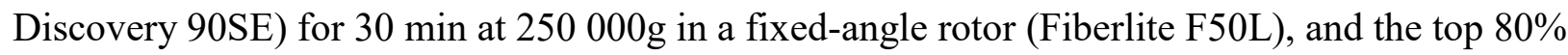
of the supernatant was extracted. The concentration was determined with a UV/vis/nIR spectrophotometer (Jasco, Tokyo, Japan) using the extinction coefficient A910 $=0.02554$ $\mathrm{L} \cdot \mathrm{mg}^{-1} \cdot \mathrm{cm}^{-1} \cdot 35$

Near-Infrared Photoluminescence Spectroscopy. Rapid two-dimensional excitation/emission photoluminescence contour maps were constructed using a procedure outlined in a previous study. ${ }^{36}$ Briefly, a supercontinuum laser (NKT SuperK Extreme EXR15) coupled to a variable bandpass filter (NKT SuperK Varia High) was used to excite DNAnanotube sample contained within a 96-well plate. The excitation wavelength varied from 500 to $827 \mathrm{~nm}$ with a $20 \mathrm{~nm}$ bandwidth and step size of $3 \mathrm{~nm}$. The emissions from the nanotube samples were collected, fed into a spectrometer with an 86 groove $/ \mathrm{mm}$ ruled grating, $320 \mathrm{~mm}$ focal length and f/4.6 aperture ratio (Princeton Instruments IsoPlane SCT 320), and finally directed into a TE-cooled InGaAs array detector (Princeton Instruments 640 X 512 pixel NIRvana: 640) with a $20 \mu \mathrm{m}$ pixel size, and Q.E. > $85 \%$ in the detection range of 0.9 to $1.7 \mu \mathrm{m}$.

In a typical experiment, $100 \mu \mathrm{L}$ of each DNA-nanotube sample were plated into a clear-bottom optical imaging 96-well plate (Corning) at a defined nanotube concentration of $5 \mathrm{mg} / \mathrm{L}$. From stock concentrations, DNA-nanotube samples were diluted in $100 \mathrm{mM} \mathrm{NaCl}$ or $100 \mathrm{mM} \mathrm{NaCl}+$ $0.1 \%$ sodium deoxycholate (SDC). The well plate was covered and incubated for 12 hours at 
room temperature in order to ensure an equilibrium in the SDC containing wells. Twodimensional excitation/emission spectroscopy was performed on all the wells with an exposure time of 0.25 or 1 second at each excitation wavelength for samples with and without SDC, respectively. All samples were examined in triplicate. Custom Labview and MATLAB codes were written to automate the data acquisition and processing, respectively.

For experiments involving the real-time addition of SDC to DNA-nanotubes, the excitation wavelength was set to either 660 or $730 \mathrm{~nm}$, with a bandwidth of $20 \mathrm{~nm}$. The emission spectra were collected in rapid succession with a constant exposure time of 0.25 seconds. After an initial time period of $\sim 10$ seconds to collect ample DNA-nanotube control data, $1 \mu \mathrm{L}$ of a stock solution of $10 \%$ SDC was spiked into the well and immediately mixed to give a final concentration of $0.1 \%$ SDC. All SDC addition experiments were performed in triplicate.

Statistical Analysis. Statistical measures and tests for correlations, in addition to exponential fitting were performed in OriginPro 8.6. PCA and k-means clustering were performed using the in-built functions in Matlab 2014a.

\section{Acknowledgments}

This work was supported in part by the NIH New Innovator Award (DP2-HD075698), the National Institute of General Medical Sciences of the NIH (P20-GM103430), the Cancer Center Support Grant (P30-CA008748), the Rhode Island Foundation (20164347), the Anna Fuller Fund, the Louis V. Gerstner Jr. Young Investigator's Fund, the Frank A. Howard Scholars Program, the Honorable Tina Brozman Foundation for Ovarian Cancer Research, Cycle for Survival, the Alan and Sandra Gerry Metastasis Research Initiative, Mr. William H. Goodwin and Mrs. Alice Goodwin and the Commonwealth Foundation for Cancer Research, the 
Experimental Therapeutics Center, the Imaging \& Radiation Sciences Program, and the Center for Molecular Imaging and Nanotechnology of Memorial Sloan Kettering Cancer Center (MSKCC). P.V.J. was supported by an NIH NCI T-32 fellowship (2T32CA062948-21). D.R. was supported by an American Cancer Society 2013 Roaring Fork Valley Research Fellowship. We thank C.Horoszko, and members of both labs, for helpful discussions.

Supporting Information. Additional data and analyses, including all fitting parameters and correlations are found in the supporting information. The Supporting Information is available free of charge on the ACS Publications website at DOI:

\section{Corresponding Author}

*roxbury@uri.edu

\section{Author Contributions}

The manuscript was written through contributions of all authors. All authors have given approval to the final version of the manuscript.

\section{References}

1. Jena, P. V.; Galassi, T. V.; Roxbury, D.; Heller, D. A., Review-Progress toward Applications of Carbon Nanotube Photoluminescence. ECS J. Solid State Sci. Technol. 2017, 6 (6), M3075-M3077.

2. Hong, G. S.; Diao, S. O.; Antaris, A. L.; Dai, H. J., Carbon Nanomaterials for Biological Imaging and Nanomedicinal Therapy. Chem. Rev. 2015, 115 (19), 10816-10906.

3. Prasek, J.; Drbohlavova, J.; Chomoucka, J.; Hubalek, J.; Jasek, O.; Adam, V.; Kizek, R., Methods for Carbon Nanotubes Synthesis-Review. J Mater. Chem. 2011, 21 (40), 15872-15884.

4. Zheng, M., Sorting Carbon Nanotubes. Top. Curr. Chem. 2017, 375 (1), 13.

5. $\quad$ Zheng, M.; Jagota, A.; Semke, E. D.; Diner, B. A.; Mclean, R. S.; Lustig, S. R.;

Richardson, R. E.; Tassi, N. G., DNA-Assisted Dispersion and Separation of Carbon Nanotubes. Nat. Mater. 2003, 2 (5), 338-342.

6. Tu, X.; Manohar, S.; Jagota, A.; Zheng, M., DNA Sequence Motifs for Structure-Specific Recognition and Separation of Carbon Nanotubes. Nature 2009, 460 (7252), 250-253. 
7. Ao, G. Y.; Streit, J. K.; Fagan, J. A.; Zheng, M., Differentiating Left- and Right-Handed Carbon Nanotubes by DNA. J. Am. Chem. Soc. 2016, 138 (51), 16677-16685.

8. Zheng, Y.; Bachilo, S. M.; Weisman, R. B., Quenching of Single-Walled Carbon Nanotube Fluorescence by Dissolved Oxygen Reveals Selective Single-Stranded DNA Affinities. J. Phys. Chem. Lett. 2017, 1952-1955.

9. $\quad$ Kruss, S.; Landry, M. P.; Vander Ende, E.; Lima, B. M. A.; Reuel, N. F.; Zhang, J. Q.; Nelson, J.; Mu, B.; Hilmer, A.; Strano, M. S., Neurotransmitter Detection Using Corona Phase Molecular Recognition on Fluorescent Single-Walled Carbon Nanotube Sensors. J. Am. Chem. Soc. 2014, $136(2), 713-724$.

10. Ulissi, Z. W.; Zhang, J. Q.; Sresht, V.; Blankschtein, D.; Strano, M. S., 2D Equation-ofState Model for Corona Phase Molecular Recognition on Single-Walled Carbon Nanotube and Graphene Surfaces. Langmuir 2015, 31 (1), 628-636.

11. Harvey, J. D.; Jena, P. V.; Baker, H. A.; Zerze, G. H.; Williams, R. M.; Galassi, T. V.; Roxbury, D.; Mittal, J.; Heller, D. A., A Carbon Nanotube Reporter of MicroRNA Hybridization Events In Vivo. Nat. Biomed. Eng. 2017, 1, 0041.

12. Kawaguchi, M.; Yamazaki, J.; Ohno, J.; Fukushima, T., Preparation and Binding Study of a Complex Made of DNA-Treated Single-Walled Carbon Nanotubes and Antibody for Specific Delivery of a "Molecular Heater" Platform. Int. J. Nanomed. 2012, 7, 4363-4371.

13. Landry, M. P.; Ando, H.; Chen, A. Y.; Cao, J.; Kottadiel, V. I.; Chio, L.; Yang, D.; Dong, J.; Lu, T. K.; Strano, M. S., Single-Molecule Detection of Protein Efflux from Microorganisms Using Fluorescent Single-Walled Carbon Nanotube Sensor Arrays. Nat. Nanotechnol. 2017, 12 (4), 368-377.

14. Roxbury, D.; Tu, X.; Zheng, M.; Jagota, A., Recognition ability of DNA for carbon nanotubes correlates with their binding affinity. Langmuir 2011, 27 (13), 8282-8293.

15. Shankar, A.; Mittal, J.; Jagota, A., Binding between DNA and carbon nanotubes strongly depends upon sequence and chirality. Langmuir 2014, 30 (11), 3176-3183.

16. Kato, Y.; Inoue, A.; Niidome, Y.; Nakashima, N., Thermodynamics on soluble carbon nanotubes: how do DNA molecules replace surfactants on carbon nanotubes? Sci. Rep. 2012, 2 , 733.

17. Cathcart, H.; Nicolosi, V.; Hughes, J. M.; Blau, W. J.; Kelly, J. M.; Quinn, S. J.;

Coleman, J. N., Ordered DNA Wrapping Switches on Luminescence in Single-Walled Nanotube Dispersions. J. Am. Chem. Soc. 2008, 130 (38), 12734-12744.

18. He, P. G.; Bayachou, M., Layer-By-Layer Fabrication and Characterization of DNAWrapped Single-Walled Carbon Nanotube Particles. Langmuir 2005, 21 (13), 6086-6092.

19. Iliafar, S.; Mittal, J.; Vezenov, D.; Jagota, A., Interaction of Single-Stranded DNA with Curved Carbon Nanotube Is Much Stronger Than with Flat Graphite. J. Am. Chem. Soc. 2014, 136 (37), 12947-12957.

20. Bergler, F. F.; Schoppler, F.; Brunecker, F. K.; Hailman, M.; Hertel, T., Fluorescence Spectroscopy of Gel-Immobilized Single-Wall Carbon Nanotubes with Microfluidic Control of the Surfactant Environment. J. Phys. Chem. C 2013, 117 (25), 13318-13323.

21. Zhao, X.; Johnson, J. K., Simulation of adsorption of DNA on carbon nanotubes. J. Am. Chem. Soc. 2007, 129 (34), 10438-10445.

22. Roxbury, D.; Mittal, J.; Jagota, A., Molecular-Basis of Single-Walled Carbon Nanotube Recognition by Single-Stranded DNA. Nano Lett. 2012, 12 (3), 1464-1469.

23. Choi, J. H.; Strano, M. S., Solvatochromism in Single-Walled Carbon Nanotubes. Appl. Phys. Lett. 2007, 90 (22). 
24. Nugraha, A. R. T.; Saito, R.; Sato, K.; Araujo, P. T.; Jorio, A.; Dresselhaus, M. S., Dielectric Constant Model for Environmental Effects on the Exciton Energies of Single Wall Carbon Nanotubes. Appl. Phys. Lett. 2010, 97 (9).

25. Larsen, B. A.; Deria, P.; Holt, J. M.; Stanton, I. N.; Heben, M. J.; Therien, M. J.; Blackburn, J. L., Effect of Solvent Polarity and Electrophilicity on Quantum Yields and Solvatochromic Shifts of Single-Walled Carbon Nanotube Photoluminescence. J. Am. Chem. Soc. 2012, 134 (30), 12485-12491.

26. Ito, M.; Ito, Y.; Nii, D.; Kato, H.; Umemura, K.; Homma, Y., The Effect of DNA Adsorption on Optical Transitions in Single Walled Carbon Nanotubes. J. Phys. Chem. C 2015, 119 (36), 21141-21145.

27. Cambre, S.; Santos, S. M.; Wenseleers, W.; Nugraha, A. R. T.; Saito, R.; Cognet, L.; Lounis, B., Luminescence Properties of Individual Empty and Water-Filled Single-Walled Carbon Nanotubes. ACS Nano 2012, 6 (3), 2649-2655.

28. Lefebvre, J., Real Time Hyperspectroscopy for Dynamical Study of Carbon Nanotubes. ACS Nano 2016, 10 (10), 9602-9607.

29. Roxbury, D.; Tu, X. M.; Zheng, M.; Jagota, A., Recognition Ability of DNA for Carbon Nanotubes Correlates with Their Binding Affinity. Langmuir 2011, 27 (13), 8282-8293.

30. Haggenmueller, R.; Rahatekar, S. S.; Fagan, J. A.; Chun, J. H.; Becker, M. L.; Naik, R. R.; Krauss, T.; Carlson, L.; Kadla, J. F.; Trulove, P. C.; Fox, D. F.; DeLong, H. C.; Fang, Z. C.; Kelley, S. O.; Gilman, J. W., Comparison of the Quality of Aqueous Dispersions of Single Wall Carbon Nanotubes Using Surfactants and Biomolecules. Langmuir 2008, 24 (9), 5070-5078.

31. O'Connell, M. J.; Bachilo, S. M.; Huffman, C. B.; Moore, V. C.; Strano, M. S.; Haroz, E. H.; Rialon, K. L.; Boul, P. J.; Noon, W. H.; Kittrell, C.; Ma, J. P.; Hauge, R. H.; Weisman, R. B.; Smalley, R. E., Band Gap Fluorescence from Individual Single-Walled Carbon Nanotubes. Science 2002, 297 (5581), 593-596.

32. Ding, C.; He, X. In K-means clustering via principal component analysis, Proceedings of the twenty-first international conference on Machine learning, ACM: 2004; p 29.

33. Polo, E.; Kruss, S., Impact of Redox-Active Molecules on the Fluorescence of PolymerWrapped Carbon Nanotubes. J. Phys. Chem. C 2016, 120 (5), 3061-3070.

34. Salem, D. P.; Landry, M. P.; Bisker, G.; Ahn, J.; Kruss, S.; Strano, M. S., Chirality Dependent Corona Phase Molecular Recognition of DNA-Wrapped Carbon Nanotubes. Carbon 2016, 97, 147-153.

35. Roxbury, D.; Jena, P.; Williams, R.; Enyedi, B.; Niethammer, P.; Marcet, S.; Verhaegen, M.; Blais-Ouellette, S.; Heller, D., Hyperspectral Microscopy of Near-Infrared Fluorescence Enables 17-Chirality Carbon Nanotube Imaging. Scientific reports 2015, 5, 14167-14167.

36. Roxbury, D.; Jena, P. V.; Shamay, Y.; Horoszko, C. P.; Heller, D. A., Cell Membrane Proteins Modulate the Carbon Nanotube Optical Bandgap via Surface Charge Accumulation. ACS Nano 2015, 10 (1), 499-506. 

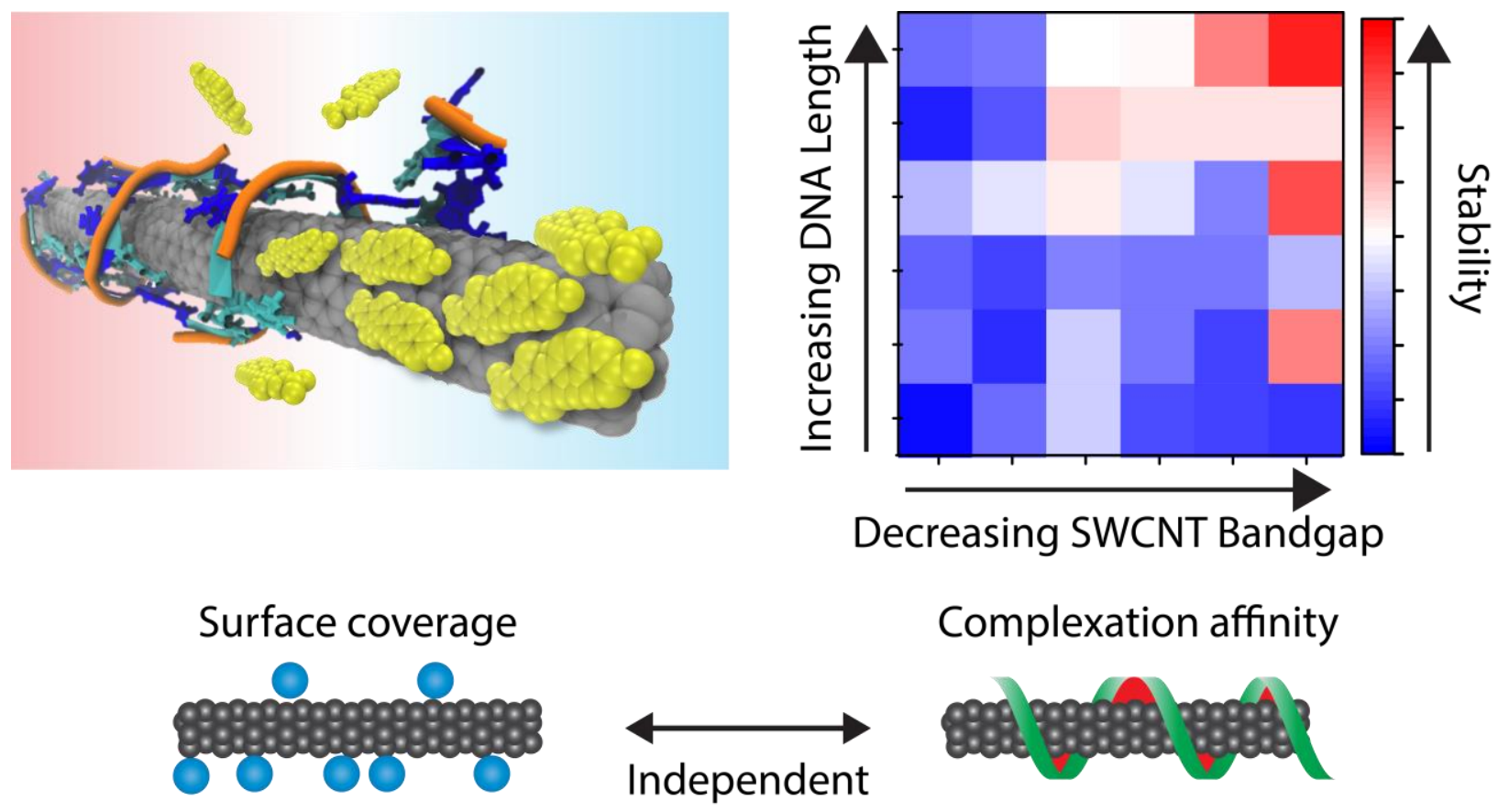

Complexation affinity

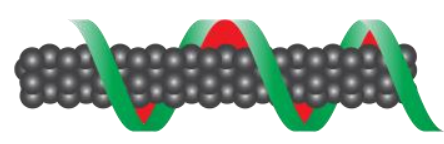

